\title{
Élaboration d'indicateurs pour l'évaluation des collectivités-amies des aînés au Canada : démarche et résultats
}

\author{
H. Orpana, Ph. D. (1,2); M. Chawla, M.P.H. (3); E. Gallagher, Ph. D. (4); E. Escaravage, M. Sc. (3)
}

Cet article a fait l'objet d'une évaluation par les pairs.

\section{Résumé}

Introduction : En 2006, l'Organisation mondiale de la Santé (OMS) a lancé le projet mondial « Villes-amies des aînés » afin de favoriser un vieillissement actif. Si un grand nombre d'initiatives " amies des aînés » ont été mises en œuvre au Canada, on dispose de peu d'information sur l'efficacité et les résultats des initiatives des collectivités-amies des aînés (CAA). En outre, les intervenants affirment qu'ils n’ont pas la capacité et les outils nécessaires pour élaborer et réaliser des évaluations relatives à leurs initiatives de CAA. Afin de pallier ces lacunes, l'Agence de la santé publique du Canada a mis au point des indicateurs pour l'évaluation des initiatives de CAA dans de nombreuses collectivités canadiennes. Ces indicateurs, destinés à répondre aux différents besoins des collectivités, ne sont pas conçus pour faire l'évaluation de répercussions collectives ou pour rendre possible une comparaison entre collectivités.

Méthodologie : Une démarche de consultation itérative fondée sur des données probantes a été employée pour l'élaboration d'indicateurs relatifs aux CAA. Elle a nécessité une revue de la littérature et une analyse du contexte. Deux rondes de consultation auprès d'experts et d'intervenants clés ont été menées, ce qui a permis de classer les indicateurs potentiels en fonction de leur importance, de leur capacité à être mis en pratique et de leur faisabilité. Une liste définitive d'indicateurs et de mesures potentielles a ensuite été mise au point, en fonction des résultats de ces consultations et de considérations clés relatives aux politiques.

Résultats : Trente-neuf indicateurs répartis en huit domaines relevant des CAA et quatre indicateurs relevant des résultats liés à la santé et aux conditions sociales à long terme ont été sélectionnés. Tous sont conformes à l'objectif énoncé, à savoir l'évaluation des initiatives de CAA à l'échelon local. Un guide convivial est disponible pour soutenir et diffuser ce travail.

Conclusion : Les indicateurs de CAA sont susceptibles d'aider les collectivités à procéder à l'évaluation de leurs initiatives amies des aînés, ce qui constitue la dernière étape du cycle des jalons pancanadiens des CAA. Les collectivités sont en effet encouragées à améliorer leurs initiatives amies des aînés à partir de l'évaluation de leurs résultats, afin d'en faire profiter un large éventail de Canadiens.

Mots-clés : amies des aînés, évaluation, vieillissement, collectivité, Canada

\section{Introduction}

En 2006, l'Organisation mondiale de la Santé (OMS) a donné le coup d'envoi au projet mondial «Villes-amies des aînés » en réunissant des représentants de villes du monde entier souhaitant favoriser un vieillissement en santé ${ }^{1}$. Cette consultation a fait ressortir huit grands domaines de la vie locale dans lesquels les collectivités pouvaient agir pour mieux répondre aux besoins des aînés : espaces extérieurs et

\section{Points saillants}

- Les collectivités ayant mis en œuvre des initiatives de collectivitésamies des aînés (CAA) sont de plus en plus nombreuses au Canada. Bon nombre d'entre elles sont prêtes à procéder à l'évaluation de leurs activités.

- L’Agence de la santé publique du Canada a établi, au moyen d'une analyse exhaustive, une liste d'indicateurs potentiels de la convivialité à l'égard des aînés susceptibles de servir à l'évaluation. Une démarche de consultation en plusieurs phases a permis de raccourcir et de peaufiner cette liste.

- La liste finale comprend 39 indicateurs relevant de huit domaines des collectivités locales et 4 indicateurs relevant des résultats liés à la santé et aux conditions sociales, tous aptes à soutenir le processus d'évaluation des collectivités.

bâtiments, transports, logement, participation au tissu social, respect et inclusion sociale, participation citoyenne et emploi, communication et information et enfin soutien communautaire et services de santé. Le Canada a été un partenaire clé lors de l'élaboration de cette approche, et quatre villes canadiennes ont pris part aux consultations ayant conduit à la conception du modèle. Le Canada comptant un nombre considérable de collectivités rurales et éloignées, les ministres responsables des aînés aux échelons fédéral, provincial et territorial ont parrainé un projet complémentaire, qui a donné lieu à la publication du document Initiative des collectivités rurales et éloignées amies des aînés : un guide².

Rattachement des auteurs :

1. Division de l'intégration scientifique, Direction des déterminants sociaux et de l'intégration scientifique, Agence de la santé publique du Canada, Ottawa (Ontario), Canada 2. École de psychologie, Université d'Ottawa, Ottawa (Ontario), Canada

3. Division de la promotion et de l'innovation en matière de santé de la population, Centre pour la promotion de la santé, Agence de la santé publique du Canada, Ottawa (Ontario), Canada 4. École des sciences infirmières (émérite), Faculté de développement humain et social, Université de Victoria, Victoria (Colombie-Britannique), Canada

Correspondance : Heather Orpana, Agence de la santé publique du Canada, 785, avenue Carling, Ottawa (Ontario) K1A 0K9; tél. : 613-878-5011; téléc. : 613-960-0944; courriel : heather.orpana@phac-aspc.gc.ca 
Dans le cadre de son rôle national de direction en matière de promotion du développement des collectivités-amies des aînés (CAA), l’Agence de la santé publique du Canada (ASPC) a défini, avec la collaboration de partenaires clés, les jalons pancanadiens des CAA. Ces jalons décrivent les étapes à suivre pour appliquer le modèle des CAA au Canada ${ }^{3}$ :

1. Établir un comité consultatif auquel des aînés participent activement;

2. Faire adopter une résolution par le conseil municipal pour soutenir et promouvoir activement les mesures visant à mettre la collectivité en voie de devenir amie des aînés;

3. Établir un plan d'action solide et concret qui corresponde aux besoins exprimés par les aînés dans la collectivité;

4. Faire la preuve de sa volonté d'agir en rendant public ce plan d'action;

5. S'engager à mesurer les activités mises en place et à évaluer et publier les résultats du plan d'action.

Plus de 900 collectivités canadiennes travaillent actuellement à devenir amies des aînés. Un certain nombre d'administrations et d'organisations non gouvernementales ont signalé ne pas avoir les capacités et les outils nécessaires pour mener à bien le cinquième jalon, celui qui consiste à réaliser des évaluations efficaces. En 2009, l'ASPC s'est réunie avec des intervenants clés et des chercheurs du domaine des collectivités-amies des aînés afin de discuter de façon globale de l'évaluation des CAA. Au cours de cette réunion, les participants ont signalé que les intervenants locaux avaient besoin d'indicateurs et de données.

En 2011, devant l'intérêt croissant manifesté par des provinces, des organisations non gouvernementales et des chercheurs canadiens, l'ASPC s'est engagée dans un processus rigoureux d'élaboration d'un ensemble d'indicateurs pour les CAA. Ces indicateurs forment un menu dans lequel les collectivités peuvent faire leur choix en fonction des enjeux locaux et de leurs capacités.

Dans cet article, nous décrivons la démarche entreprise par l'ASPC, les motifs et les principes qui sous-tendent le projet des indicateurs des CAA et les progrès accomplis jusqu'à présent dans la définition des indicateurs des CAA.

\section{Méthodologie et résultats}

\section{Détermination des indicateurs et classement par ordre de priorité}

La démarche qu'a employée l'ASPC afin de mettre au point cet ensemble d'indicateurs a été adaptée de méthodes fiables ayant précédemment servi à l'élaboration d'autres cadres d'indicateurs en santé publique ${ }^{4-6}$. De façon générale, l'élaboration d'indicateurs consiste à définir le but qui leur est associé, à créer un cadre conceptuel sur la base de fondements théoriques, de politiques ou de données ${ }^{4}$ et à sélectionner ou créer les indicateurs eux-mêmes ${ }^{5}$.

En l'occurrence, la démarche d'élaboration d'indicateurs a visé à mettre au point un menu d'indicateurs qui permette aux collectivités qui entreprennent des initiatives amies des aînés de choisir des indicateurs adaptés à leurs activités d'évaluation et de surveillance. Le cadre relatif aux villesamies des aînés de l'OMS a fourni le cadre conceptuel global, notamment en ce qui concerne les huit domaines autour desquels les collectivités peuvent axer leurs interventions en vue d'aider leurs résidents à vieillir en santé ${ }^{1}$.

Un Groupe de travail sur les indicateurs des collectivités-amies des aînés a été mis sur pied pour encadrer la réalisation du projet des indicateurs des CAA. Ce groupe de travail, qui s'est réuni régulièrement tout au long du projet, était composé de représentants de l'ASPC, des gouvernements provinciaux et territoriaux, des municipalités et d'organisations non gouvernementales, de chercheurs et d'aînés.

\section{Revue de la littérature et analyse du contexte}

La première étape a consisté à trouver des indicateurs potentiels au moyen d'une revue de la littérature. Nous avons cherché, dans les bases de données suivantes, les articles ayant fait l'objet d'une évaluation par les pairs et publiés entre 1990 et 2012 : Web of Science, AgeLine, registre d'essais Cochrane Injuries Group, CINAHL Database, MEDLINE, Health Source et Social Sciences Citation Index. Seules les publications en anglais et en français concernant les adultes de 65 ans et plus ont été retenues. La recherche a été faite au moyen des termes suivants et de combinaisons de ceux-ci : personnes âgées, aînés, évaluation, villes/collectivités/entreprises/hôpitaux amis des aînés, collectivités adaptées aux besoins des aînés, collectivités amies des personnes âgées, logements visitables, environnement bâti, logement, modification de domicile, codes du bâtiment, escaliers, trottoirs, transport, environnement social/inclusion sociale, respect, emploi, bénévolat, résultats, amélioration, indicateurs de réussite, chutes, accidents, accidents d'automobile, accidents de la route, communication, soutien communautaire et services de santé.

En premier lieu, nous avons évalué la pertinence des titres. Les articles qui ne correspondaient de toute évidence pas à l'objet de la recherche (comme les articles sur les soins cliniques associés à une maladie particulière) ont été écartés à cette étape. Nous avons ensuite analysé les résumés des articles restants et nous avons inclus ceux qui portaient directement ou indirectement sur les indicateurs ou les mesures se rapportant aux collectivités-amies des aînés. La plupart de ces articles traitaient principalement des évaluations de processus ou des résultats à court terme du travail réalisé par les collectivités. Nous n'avons trouvé aucun compte rendu d'évaluation des résultats à long terme d'initiatives amies des aînés.

Sur la liste initiale de plus de 2000 documents, nous avons jugé pertinents 23 articles.

Nous avons également réalisé une analyse des activités d'évaluation des CAA déjà menées au Canada et à l'étranger. Nous avons communiqué par courriel avec les principaux intervenants dans les provinces, les territoires et certaines municipalités au sujet de leurs activités, et nous leur avons demandé de fournir des exemplaires de leurs outils d'évaluation. Les mots clés " ami des aînés » et " évaluation » ont servi à trouver, à l'aide du moteur de recherche Google, les programmes en usage ailleurs dans le monde et les documents pertinents de la littérature grise. Nous avons conservé les documents trouvés au cours de cette recherche Internet s'ils contenaient soit de l'information sur les initiatives amies des aînés ou les évaluations locales existantes, soit des renseignements sur les indicateurs ou leurs mesures : 5 rapports communautaires ont ainsi été ajoutés ${ }^{7-11}$. Un examen de nos dossiers a permis de trouver 20 documents additionnels satisfaisant également à nos critères. 
Nous avons ainsi retenu au total 43 documents et articles pour évaluation. Douze provenaient des États-Unis ${ }^{12-23}, 3$ relevaient d'études portant sur plusieurs pays ${ }^{1,24,25}$, 2 portaient sur la France ${ }^{26,27}, 2$ sur l'Australie $^{28,29}, 2$ sur les Pays-Bas ${ }^{30,31}$ et 1 sur le Royaume-Uni ${ }^{32}$. Pour le Canada, 5 documents provenaient de la ColombieBritannique $^{33-37}, 5$ du Québec $^{7,38-41}, 4$ de l'Ontario ${ }^{9,10,42,43}, 1$ du Manitoba ${ }^{8}, 1$ de la Saskatchewan $^{11}$ et 5 avaient une portée nationale $e^{4-48}$.

Deux spécialistes en recherche qualitative ont analysé les articles et rapports et ont rédigé, d'après le contenu des articles, des notes détaillées sur les indicateurs potentiels mesurant l'adaptation aux besoins des aînés, et ce, pour chacun des huit domaines déterminés par l'OMS ${ }^{1}$. Cette méthode de collecte de données qualitatives sur une base théorique, ou déductive ${ }^{49}$, a permis d'établir une liste initiale de 241 indicateurs potentiels. Les deux chercheurs ont défini conjointement plusieurs sous-thèmes à l'intérieur de chaque domaine en regroupant les indicateurs similaires et en leur assignant des codes (ou étiquettes) reflétant les concepts sous-jacents communs (tableau 1). Il s'agit là d'un exemple de codage inductif, ou guidé par les données $^{49}$. L'équipe de recherche (qui comptait quatre membres spécialisés en méthodes qualitatives ou quantitatives, en gérontologie et en CAA) a passé en revue la liste d'indicateurs potentiels afin d'en évaluer la redondance, la pertinence et la clarté. D'un commun accord, ils ont regroupé certains concepts en un seul indicateur et ont éliminé les concepts jugés non pertinents, faisant ainsi passer la liste à 194 indicateurs. Quelques indicateurs, quoique considérés comme importants du point de vue du vieillissement en santé, et initialement inscrits dans les huit domaines, coïncidaient mal avec les sous-thèmes : ils ont été regroupés dans un nouveau domaine, celui des "résultats liés à la santé et aux conditions sociales ». Cette structure a été présentée au Groupe de travail sur les indicateurs des collectivités-amies des aînés pour approbation.

Nous avons également mis au point, à partir d'une revue de la littérature, une liste de critères potentiels et nous l'avons présentée au Groupe de travail sur les indicateurs des collectivités-amies des aînés : fondé sur des données probantes, reflétant un fardeau, représentatif, disponible, pouvant faire l'objet de changements, compréhensible, reproductible, important, sensé, viable, direct, objectif, utile, attribuable, pratique et adéquat ${ }^{50-53}$. L'équipe de recherche a établi que les critères les plus importants pour la sélection des indicateurs étaient leur importance/pertinence, leur capacité à être mis en pratique et leur faisabilité, ce qui a été confirmé par le Groupe de travail.

\section{Établissement de l'ordre de priorité des indicateurs par la mobilisation et la consultation des intervenants}

La deuxième phase de la démarche de sélection des indicateurs a consisté en deux consultations menées, au moyen d'un outil de sondage en ligne élaboré à l'aide de FluidSurvey, auprès d'intervenants ciblés, dans le but de réduire le nombre d'indicateurs potentiels.

Consultation $n^{o} \mathbf{1}$ : La première consultation ciblait 789 intervenants reconnus, notamment des représentants provinciaux et territoriaux, des représentants municipaux, des membres d'organisations non gouvernementales, des chercheurs et le personnel de l'équipe de projet ou des bénévoles travaillant à des projets de collectivités-amies des aînés. Nous avons sélectionné ces intervenants au moyen de listes de coordonnées établies par le Groupe de référence sur les collectivitésamies des aînés et le Groupe de travail sur les indicateurs des collectivités-amies des aînés. Les répondants ont classé les 194 indicateurs potentiels selon leur importance dans la mesure de la convivialité des villes à l'égard des aînés, leur attribuant une note sur une échelle de 1 à 4,1 signifiant "pas important », 2 "peu important», 3 « important » et 4 « très important».

En tout, 191 personnes ont répondu à ce premier sondage de consultation (taux de réponse de $24 \%$ ). Toutes les provinces ont été représentées à l'exception du NouveauBrunswick, mais les effectifs de répondants étaient faibles à l'Île-du-Prince-Édouard et en Saskatchewan. Aucun répondant ne provenait des territoires. Tous les groupes d'intervenants ciblés étaient représentés. La majorité des répondants étaient des femmes (70 \%), ont répondu en anglais (61\%) et avaient moins de 55 ans (59\%). Les intervenants habitaient dans des collectivités de tailles diverses : $65 \%$ d'entre eux vivaient dans des agglomérations de 1000 à 29999 habitants, 9 \% dans des agglomérations de 30000 à 99999 habitants et $23 \%$ dans des agglomérations de 100000 habitants et plus.
Le tableau 2 présente les caractéristiques des répondants des première et deuxième consultations.

Les notes moyennes attribuées à l'importance des différents indicateurs (sur une échelle de 1 à 4) se situaient entre 2,58 et 3,69 . Sur les 5 indicateurs ayant obtenu la plus haute note, 3 concernaient l'amélioration des services de santé et communautaires :

1. Existence de programmes de soutien destinés aux aidants (3,69 sur une échelle de 1 à 4) (services de santé de soutien)

2. Disponibilité de programmes de repas à prix modique $(3,67)$ (services communautaires)

3. Niveau des besoins de soins à domicile non comblés $(3,66)$ (services communautaires)

4. Existence de règlements ou de normes pour les foyers de soins infirmiers $(3,64)$ (services communautaires)

5. Nombre d'options de transport abordables $(3,64)$ (options de transport et transport en commun).

Les indicateurs classés parmi les $50 \%$ plus importants de chaque sous-domaine (en fonction de leur note moyenne) ont été retenus pour une analyse plus poussée. Les données ont également été stratifiées selon la taille de la collectivité afin de pouvoir examiner séparément les données des collectivités de taille petite ou moyenne et celles des collectivités de grande taille. Dans les rares cas où les indicateurs dont la note d'importance moyenne se situait dans les $50 \%$ supérieurs n'étaient pas les mêmes dans les collectivités de taille petite ou moyenne et dans celles de grande taille, nous avons ajouté des indicateurs afin d'inclure les $50 \%$ supérieurs pour les deux groupes. Cette démarche a permis de réduire à 129 le nombre d'indicateurs. Nous avons ensuite combiné certains indicateurs se rapportant à des concepts similaires, ce qui a fait passer la liste à 109 indicateurs.

Sur les 191 personnes ayant répondu au premier sondage de consultation, 93 ont indiqué qu'elles aimeraient participer à un sondage de consultation subséquent.

Consultation $n^{o} 2$ : Le second sondage de consultation avait comme objectif de réduire la liste des 109 indicateurs potentiels en fonction de leur " capacité à être 
TABLEAU 1

Indicateurs potentiels et sous-thèmes pour l'évaluation des collectivités-amies des aînés au Canada

\begin{tabular}{|c|c|c|c|}
\hline Domaine & Sous-thèmes initiaux & $\begin{array}{c}\text { Nombre initial d'indicateurs } \\
\text { potentiels }\end{array}$ & Nombre final d'indicateurs ${ }^{a}$ \\
\hline Espaces extérieurs et bâtiments & $\begin{array}{l}\text { Potentiel piétonnier } \\
\text { Accessibilité réelle et perçue } \\
\text { Blessures } \\
\text { Prévention du crime }\end{array}$ & 53 & $\begin{array}{l}7 \\
\text { (4 indicateurs ont obtenu un degré d'accord } \\
\text { élevé et étaient souvent employés dans la } \\
\text { littérature; } 3 \text { indicateurs ont obtenu un degré } \\
\text { d'accord moyen et étaient souvent employés) }\end{array}$ \\
\hline Transports & $\begin{array}{l}\text { Options de transport et transport } \\
\text { en commun } \\
\text { Rues et stationnements } \\
\text { conviviaux à l'égard des aînés }\end{array}$ & 28 & $\begin{array}{l}6 \\
\text { (1 indicateur a obtenu un degré d'accord élevé } \\
\text { et était souvent employé; } 5 \text { indicateurs ont } \\
\text { obtenu un degré d'accord moyen et étaient } \\
\text { souvent employés; } 1 \text { indicateur avait un faible } \\
\text { degré d'accord, mais était souvent employé et } \\
\text { concordait avec les indicateurs de l'OMS; } 2 \text { de } \\
\text { ces indicateurs ont été regroupés par la suite) }\end{array}$ \\
\hline Participation au tissu social & $\begin{array}{l}\text { Participation à des activités } \\
\text { sociales } \\
\text { Occasions de participation } \\
\text { Accessibilité des occasions de } \\
\text { participation }\end{array}$ & 24 & $\begin{array}{c}6 \\
\text { (4 indicateurs ont obtenu un degré d'accord } \\
\text { élevé et étaient souvent employés; } 2 \\
\text { indicateurs ont obtenu un degré d'accord } \\
\text { moyen et étaient souvent employés) }\end{array}$ \\
\hline Respect et inclusion sociale & $\begin{array}{l}\text { Existence d'activités intergénéra- } \\
\text { tionnelles } \\
\text { Sentiment d'appartenance }\end{array}$ & 13 & $\begin{array}{c}2 \\
\text { (tous deux ont obtenu un degré d'accord élevé) }\end{array}$ \\
\hline $\begin{array}{l}\text { Soutien communautaire et services de } \\
\text { santé }\end{array}$ & $\begin{array}{l}\text { Médecin de famille } \\
\text { Services de santé de soutien } \\
\text { Services communautaires }\end{array}$ & 30 & $\begin{array}{l}5 \\
\text { (4 indicateurs ont obtenu un degré d'accord } \\
\text { élevé et étaient souvent employés; } 1 \text { indicateur } \\
\text { a obtenu un degré d'accord moyen et était } \\
\text { souvent employé) }\end{array}$ \\
\hline $\begin{array}{l}\text { Résultats liés à la santé et aux conditions } \\
\text { sociales des aînés }\end{array}$ & & 4 & 4 \\
\hline Total & & 241 & 43 \\
\hline
\end{tabular}

Abréviation : OMS, Organisation mondiale de la Santé.

Remarque : Les huit premiers domaines de la convivialité à l'égard des aînés correspondent à ceux déterminés par l'Organisation mondiale de la Santé1. L'équipe de recherche a ajouté les « résultats liés à la santé et aux conditions sociales » afin de représenter les résultats à long terme d'une collectivité-amie des aînés.

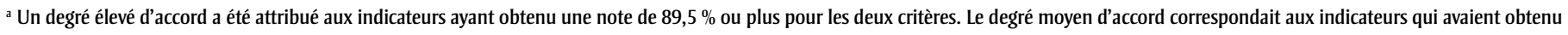

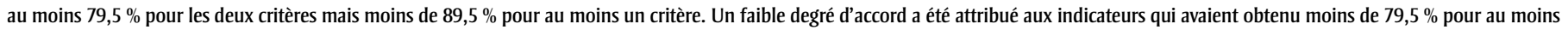

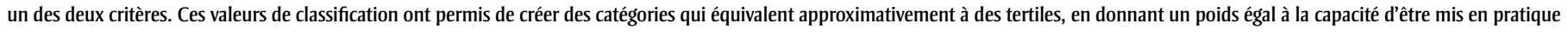
et à la faisabilité. 
TABLEAU 2

Caractéristiques des répondants aux sondages de consultation $\mathbf{n}^{0} 1$ et $\mathbf{n}^{0} \mathbf{2}$ ayant servi à la sélection d'indicateurs pour l'évaluation des collectivités-amies des aînés au Canada

\begin{tabular}{|c|c|c|}
\hline \multirow[t]{2}{*}{ Caractéristiques } & \multicolumn{2}{|c|}{ Proportion des répondants (\%) } \\
\hline & $\begin{array}{c}\text { Consultation } \mathrm{n}^{0} 1 \\
(\mathrm{n}=191)\end{array}$ & $\begin{array}{c}\text { Consultation } n^{0} 2 \\
(n=49)\end{array}$ \\
\hline \multicolumn{3}{|l|}{ Sexe } \\
\hline Femme & 70 & 80 \\
\hline Homme & 29 & 17 \\
\hline Non indiqué & 2 & 2 \\
\hline Moins de 55 & 59 & 37 \\
\hline 55 à 64 & 28 & 35 \\
\hline 65 à 74 & 11 & 17 \\
\hline 75 et plus & 1 & 7 \\
\hline Préfère ne pas répondre & 2 & 4 \\
\hline \multicolumn{3}{|l|}{ Groupe d'intervenants ${ }^{\mathrm{a}}$} \\
\hline Aîné (55 ans ou plus) & 36 & 45 \\
\hline Membre d'une famille comptant un aîné ou aidant d'un aîné & 21 & 21 \\
\hline Fournisseur de services de santé ou de services sociaux & 11 & 23 \\
\hline Fournisseur de services de transport, de services de logement ou d'autres services communautaires & 13 & 17 \\
\hline Décideur ou responsable de l'élaboration des politiques & 17 & 28 \\
\hline Éducateur ou chercheur & 11 & 23 \\
\hline Employé municipal & 45 & 32 \\
\hline Représentant élu & 11 & 11 \\
\hline Membre ou dirigeant d'un groupe de planification communautaire favorable aux aînés & - & 47 \\
\hline Préfère ne pas répondre & 1 & 2 \\
\hline \multicolumn{3}{|l|}{ Province ou territoire } \\
\hline Terre-Neuve-et-Labrador & 10 & 9 \\
\hline Île-du-Prince-Édouard & 1 & - \\
\hline Nouvelle-Écosse & 12 & 6 \\
\hline Québec & 39 & 13 \\
\hline Ontario & 10 & 23 \\
\hline Manitoba & 9 & 13 \\
\hline Saskatchewan & 1 & 2 \\
\hline Alberta & 5 & 9 \\
\hline Colombie-Britannique & 13 & 26 \\
\hline \multicolumn{3}{|l|}{ Taille de la collectivité } \\
\hline Petite agglomération, regroupant entre 1000 et 29999 habitants & 65 & 51 \\
\hline Agglomération moyenne, regroupant entre 30000 et 99999 habitants & 9 & 15 \\
\hline Grande agglomération, regroupant au moins 100000 habitants & 23 & 32 \\
\hline Préfère ne pas répondre & 3 & 2 \\
\hline
\end{tabular}

Remarque : Les chiffres étant arrondis, il se peut que la somme des pourcentages ne soit pas égale à $100 \%$.

${ }^{\text {a }}$ Les répondants pouvaient faire partie de plusieurs groupes d'intervenants. 
mis en pratique » et de leur " faisabilité », conformément aux définitions données ci-dessous.

Pour qu'un indicateur ait la capacité d'être mis en pratique, il doit être soumis à l'influence de la collectivité locale ou régionale, du gouvernement ou du secteur privé et subir des modifications en réponse à une action. Ce critère a été utilisé à la réunion du groupe chargé de l'élaboration d'indicateurs pour les villes-amies des aînés à l'échelle mondiale ${ }^{50}$ ainsi que par l'Équipe de développement des indicateurs de blessure au Canada ${ }^{51}$. Il rejoint également la notion de capacité de changement (changeabily) de Daniel ${ }^{52}$.

Pour qu'un indicateur obtienne sa mention de " faisabilité », il faut que les données qui s'y rapportent soient mesurables (p. ex. données administratives ou provenant d'un sondage) ou descriptibles (p. ex. photo ou histoire) de manière réaliste, sans obstacle à leur collecte ou à leur utilisation. Ces données doivent être utilisables pour enrichir et rendre concrets les résultats d'un programme ${ }^{53}$. Les méthodes de collecte de données doivent être simples et réalistes ${ }^{52}$.

En raison de la lassitude observée chez les répondants lors du premier sondage, les choix de réponse du second sondage ont été restreints à " oui ", " non » et " je ne sais pas ou je n'ai pas d'opinion ». Deux questions portant sur les méthodes favorites de collecte de données et la conception d'un guide produit par l'ASPC ont aussi été incluses dans cette consultation.

Ce second sondage a été transmis, au moyen de la plateforme de sondage en ligne, aux 93 participants du premier sondage intéressés à participer à une consultation subséquente et 49 d'entre eux y ont répondu (taux de réponse de $52 \%$ ). Comme l'illustre le tableau 2, $80 \%$ des répondants étaient des femmes, et $92 \%$ d'entre eux ont répondu en anglais. La plupart des régions du Canada et un vaste éventail de groupes d'intervenants étaient représentés.

Nous avons calculé la proportion de répondants approuvant la faisabilité d'un indicateur donné et sa capacité à être mis en pratique. Trois catégories ont été créées afin de regrouper les indicateurs selon ces deux critères : un degré élevé d'accord pour ceux ayant obtenu une note de
$89,5 \%$ ou plus pour les deux critères, un degré moyen d'accord pour ceux ayant obtenu $79,5 \%$ ou plus pour les deux critères mais moins de $89,5 \%$ pour l'un des deux et enfin un faible degré d'accord pour ceux ayant obtenu moins de 79,5\% pour au moins un des deux critères. Ces valeurs de classification ont permis de créer des catégories équivalant approximativement à des tertiles et accordant un poids égal à la capacité d'être mis en pratique et à la faisabilité.

$\mathrm{Au}$ total, 38 indicateurs ont obtenu un degré élevé d'accord, 47 indicateurs, un degré moyen d'accord et 24 indicateurs, un faible degré d'accord.

\section{Indicateurs}

Nous avons établi la liste définitive d'indicateurs (tableau 1) en combinant le degré d'accord, la fréquence des références au concept dans les publications évaluées par les pairs et dans la littérature grise, la concordance perçue avec les indicateurs proposés par l'OMS et le fait que l'indicateur soit ou non une mesure d'impact.

Les quatre indicateurs de résultats liés à la santé et aux conditions sociales à long terme ont tous été retenus, en raison de leur concordance avec le cadre alors proposé par l'OMS. Une liste définitive de 43 indicateurs a ainsi été obtenue. Ces indicateurs sont présentés dans le tableau 3 par domaine et par thème à l'intérieur de chaque domaine.

Les répondants ont également été invités à faire part de leurs suggestions au sujet de l'élaboration d'un guide ou d'un outil d'évaluation de leurs initiatives amies des aînés. Les thèmes suivants sont ressortis de l'analyse de leurs réponses :

- L'outil doit inclure des définitions et des critères clairs pour mesurer les indicateurs, il doit demeurer souple et s'adapter aux caractéristiques de chaque collectivité et il doit également intégrer le plus possible de mesures du comportement.

- Les résultats doivent conduire à agir à l'échelle locale.

- Des résultats à long terme doivent être intégrés.
- Le coût des évaluations ne devrait pas constituer un obstacle (ce qui soulève la question de la capacité des collectivités, sur le plan des ressources humaines et financières, à mener des évaluations).

Dans le second sondage de consultation, les répondants étaient invités à formuler des suggestions sur les types de méthodes de collecte de données les plus pratiques pour l'évaluation des initiatives amies des aînés. Moins de 60 \% d'entre eux ont dit considérer les méthodes suivantes comme étant « très pratiques » ou " pratiques » : entrevues en personne (54\%), entrevues téléphoniques (51\%), récits (58\%) et photographies ou enregistrements vidéo (56\%). Toutes les autres méthodes ont été considérées comme étant " très pratiques » ou "pratiques » par au moins $75 \%$ des répondants (questionnaire en ligne, questionnaire sur papier, observations et vérifications, information recueillie à d'autres fins, données administratives, recours à l'analyse de données secondaires). Les méthodes jugées les plus pratiques ont été les discussions en groupe (100\%), les questionnaires sur papier (91 \%) et l'utilisation de données administratives (87\%).

Les résultats des deux consultations ont été synthétisés, traduits en français et diffusés à l'ensemble des participants.

À partir de l'information fournie par les sondages de consultation et les conseils formulés par le Groupe de référence sur les collectivités-amies des aînés, on a défini un éventail de mesures susceptibles de constituer des outils pour les collectivités souhaitant inclure des indicateurs de convivialité à l'égard des aînés dans leurs activités d'évaluation. On a associé à chaque indicateur une ou plusieurs des cinq méthodes de mesure potentielles : outils d'évaluation, outils d'accessibilité, données disponibles, inventaires de programmes et enquêtes. Des méthodes qualitatives comme quantitatives ont été incluses. Des outils et des données disponibles ont été répertoriés à l'intention des collectivités souhaitant évaluer leurs activités. Par la suite, un guide convivial, le Guide d'évaluation pour les collectivitésamies des aînés (disponible sur le site de l'ASPC à http://www.phac-aspc.gc.ca /seniors-aines/indicators-indicateurs-fra .php), a été élaboré afin de faire connaître aux collectivités intéressées les divers 
TABLEAU 3

Liste d'indicateurs pour les collectivités-amies des aînés

\begin{tabular}{|c|c|c|c|}
\hline \multicolumn{2}{|c|}{ Domaine } & \multirow{2}{*}{$\begin{array}{l}\text { Thème } \\
\text { Potentiel piétonnier }\end{array}$} & \multirow[b]{2}{*}{$\begin{array}{l}\text { Indicateur } \\
\text { 1. Nombre d'aires de repos et distance entre elles. } \\
\text { 2. Nombre de toilettes accessibles. } \\
\text { 3. Passages piétonniers sécuritaires (p. ex. temps adéquat pour traverser, passages piétonniers en sections } \\
\text { courantes, haltes aux terre-pleins d'intersection, bonne visibilité). } \\
\text { 4. Trottoirs, sentiers et voies piétonnières existants et en bon état (p. ex. largées, bien éclairés et exempts de } \\
\text { glace et de neige, surfaces régulières, rampes d'accès, voies cyclables distinctes). }\end{array}$} \\
\hline 1 & $\begin{array}{l}\text { Espaces } \\
\text { extérieurs et } \\
\text { bâtiments }\end{array}$ & & \\
\hline & & Accessibilité réelle et perçue & $\begin{array}{l}\text { 5. Les bâtiments publics disposent d'accès adéquats et la circulation à proximité des bâtiments est facile (p. ex. } \\
\text { porte d'accès au rez-de-chaussée, entrée à niveau, rampes d'accès pour fauteuils roulants, portes automa- } \\
\text { tiques, couloirs assez larges pour permettre le passage de scooters et de fauteuils roulants). }\end{array}$ \\
\hline & & Blessures & 6. Nombre de chutes et autres blessures subies par des aînés (dans des lieux publics). \\
\hline & & Prévention du crime & $\begin{array}{l}\text { 7. Disponibilité de stratégies, de cours et de programmes de prévention du crime s'adressant aux aînés (visant } \\
\text { notamment la fraude et la violence envers les aînés). }\end{array}$ \\
\hline \multirow[t]{2}{*}{2} & Transports & $\begin{array}{l}\text { Options de transport et } \\
\text { transport en commun }\end{array}$ & $\begin{array}{l}\text { 8. Disponibilité d'une variété de services de transport abordables (p. ex. partenariats publics/privés, } \\
\text { programme de conducteurs bénévoles, « parc-o-bus », " stationnez et partez », navettes). } \\
\text { 9. Proportion (ou nombre) d'autobus accessibles et propres, dont la destination et le numéro sont clairement } \\
\text { indiqués. } \\
\text { 10. Arrêts d'autobus et abribus sécuritaires et faciles d'accès (p. ex. comportant des places assises, bien } \\
\text { éclairés, recouverts, déneigés, à proximité des résidences pour aînés). } \\
\text { 11. Proportion de personnes âgées de } 65 \text { ans ou plus qui ont accès au transport en commun et qui l'utilisent. }\end{array}$ \\
\hline & & $\begin{array}{l}\text { Rues et stationnements } \\
\text { conviviaux à l'égard des aînés }\end{array}$ & $\begin{array}{l}\text { 12. La signalisation et le marquage des rues sont clairs et appropriés. } \\
\text { 13. Les parcs et les espaces de stationnement sont déglacés et déneigés. }\end{array}$ \\
\hline \multirow[t]{4}{*}{3} & Logement & Disponibilité en logement & $\begin{array}{l}\text { 14. Disponibilité de logements abordables qui sont bien conçus, bien construits, bien situés et sécuritaires, et } \\
\text { pour lesquels les temps d'attente sont courts. } \\
\text { 15. Disponibilité d'options de logements multifonctionnels et d'options de logements pour vieillir chez soi qui } \\
\text { sont abordables. }\end{array}$ \\
\hline & & $\begin{array}{l}\text { Programmes de logement et } \\
\text { ressources }\end{array}$ & $\begin{array}{l}\text { 16. Disponibilité de programmes visant à améliorer l'accès, la sécurité et les possibilités d'adaptation des } \\
\text { logements (p. ex. mains courantes, rampes, détecteurs de fumée). } \\
\text { 17. Existence d'une ressource dressant la liste des services d'entretien de logement, des services de soutien et } \\
\text { des services de soins à domicile pour aînés. }\end{array}$ \\
\hline & & Capacité de vieillir chez soi & $\begin{array}{l}\text { 18. Proportion de personnes âgées de } 65 \text { ans ou plus qui désirent demeurer dans leur résidence actuelle et qui } \\
\text { sont confiantes qu'elles auront les moyens financiers de le faire. }\end{array}$ \\
\hline & & $\begin{array}{l}\text { Informations sur le soutien } \\
\text { au logement }\end{array}$ & $\begin{array}{l}\text { 19. Connaissance des programmes de suppléments au loyer ou autres programmes s'adressant aux aînés } \\
\text { (p. ex. prêts au logement). }\end{array}$ \\
\hline \multirow[t]{3}{*}{4} & $\begin{array}{l}\text { Participation } \\
\text { au tissu } \\
\text { social }\end{array}$ & $\begin{array}{l}\text { Participation à des activités } \\
\text { sociales }\end{array}$ & $\begin{array}{l}\text { 20. Proportion de personnes âgées de } 65 \text { ans ou plus qui participent à des activités sociales au moins une fois } \\
\text { par semaine (p. ex. rencontres avec amis ou voisins; activités civiques, spirituelles ou culturelles; travail ou } \\
\text { bénévolat). }\end{array}$ \\
\hline & & Occasions de participation & $\begin{array}{l}\text { 21. Disponibilité de programmes d'apprentissage ou de loisirs s'adressant spécifiquement aux aînés (p. ex. } \\
\text { cours d'informatique, jardins communautaires, artisanat, jeux, cours d'activité physique). } \\
\text { 22. Disponibilité de programmes de loisirs et de programmes sociaux intergénérationnels. } \\
\text { 23. Possibilités de s'engager avec des personnes de tous âges par l'entremise d'activités sociales, culturelles, } \\
\text { spirituelles et de loisirs. } \\
\text { 24. Programmes de loisirs pour aînés qui sont abordables. }\end{array}$ \\
\hline & & $\begin{array}{l}\text { Accessibilité des occasions de } \\
\text { participation }\end{array}$ & $\begin{array}{l}\text { 25. Les lieux publics où se déroulent les activités communautaires sont accessibles (p. ex. toilettes adaptées, } \\
\text { rampe d'accès, bon éclairage, contrôle de la température). }\end{array}$ \\
\hline \multirow[t]{2}{*}{5} & $\begin{array}{l}\text { Respect et } \\
\text { inclusion }\end{array}$ & $\begin{array}{l}\text { Existence d'activités } \\
\text { intergénérationnelles }\end{array}$ & 26. Existence d'activités familiales intergénérationnelles. \\
\hline & sociale & Sentiment d'appartenance & 27. Niveau de sentiment d'appartenance à la collectivité. \\
\hline \multirow[t]{3}{*}{6} & Participation & Chômage et emploi & 28. Taux de chômage et d'emploi parmi les aînés. \\
\hline & $\begin{array}{l}\text { citoyenne et } \\
\text { emploi }\end{array}$ & Formation et soutien & $\begin{array}{l}\text { 29. Existence de soutien aux bénévoles (p. ex. formation, transport, remboursement des dépenses, témoignagé } \\
\text { de reconnaissance). } \\
\text { 30. Disponibilité de formations visant l'adaptation du milieu de travail pour répondre aux besoins des aînés. }\end{array}$ \\
\hline & & Accessibilité & 31. Les assemblées et les immeubles municipaux sont faciles d'accès. \\
\hline
\end{tabular}

Suite page suivante 
TABLEAU 3 (suite)

Liste d'indicateurs pour les collectivités-amies des aînés

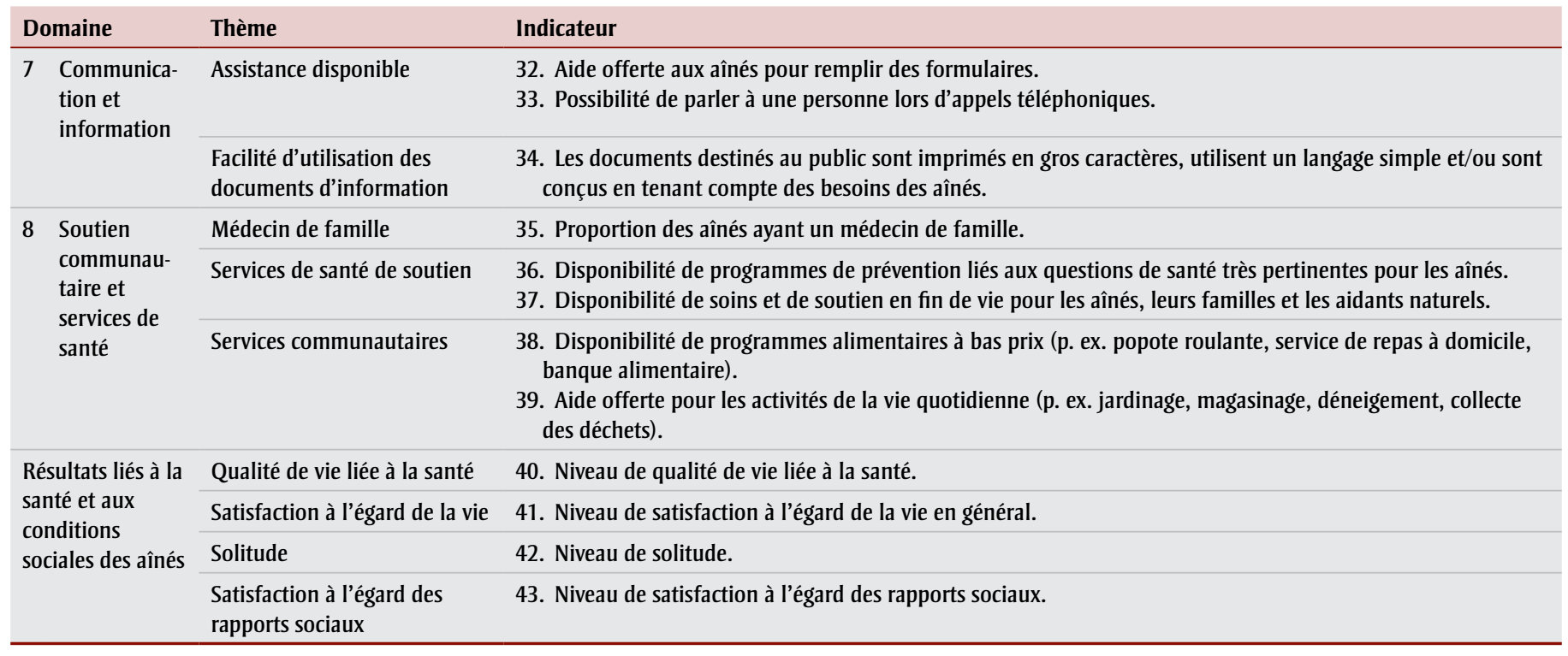

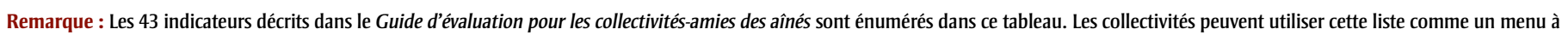
partir duquel elles sélectionnent les indicateurs les plus pertinents pour leurs activités amies des aînés.

indicateurs, les méthodes de mesure suggérées et les outils utiles.

\section{Analyse}

Notons que les listes initiales d'indicateurs ont été fondées sur la littérature sur les CAA disponible en 2012, au moment de l'enclenchement du processus. Il apparaissait alors clairement que les publications évaluées par les pairs et la littérature grise au sujet des CAA présentaient les limites suivantes : attention insuffisante portée aux populations spéciales (groupes ethniques, membres des Premières Nations et personnes lesbiennes, gaies, bisexuelles, transgenres ou en questionnement [LGBTQ]), recours massif aux groupes de discussion pour obtenir une rétroaction de la collectivité (ce qui peut désavantager les personnes ayant des problèmes de mobilité), absence de prise en compte des environnements spécialisés (dont les centres d'hébergement et de soins de longue durée et les hôpitaux) et faible accent mis sur l'intégration et la cohésion familiale en tant qu'éléments essentiels de la convivialité des villes à l'égard des aînés.

Depuis lors, la littérature sur les CAA s'est considérablement développée. Comme nous l'avons mentionné dans l'introduction, l'OMS a entrepris une démarche en vue de déterminer des indicateurs de base à l'intention du Réseau mondial des villes et des communautés amies des aînés en 2011, peu après le lancement par l'ASPC du projet décrit dans cet article, et elle a publié un guide en $2015^{54}$. Les indicateurs proposés par l'OMS sont considérablement proches de ceux proposés par l'ASPC, ce qui n'est pas étonnant dans la mesure où les deux projets ont pris appui l'un sur l'autre et ont été élaborés en parallèle. Leurs concepts communs sont notamment le potentiel piétonnier, l'accessibilité des lieux publics et des immeubles, du transport en commun et des véhicules ainsi que des arrêts des véhicules de transport en commun, la disponibilité en logements abordables, la participation à des activités bénévoles, à un travail rémunéré et à des activités socioculturelles, la disponibilité d'information, de services de santé et de services sociaux et enfin la qualité de vie. Dans une synthèse réaliste récente de la convivialité concrète des villes à l'égard des aînés du Réseau européen des villes-santé, les huit domaines de l'OMS ont été répartis en trois groupes : environnement physique, environnement social et services municipaux ${ }^{55}$. Les chercheurs ont synthétisé de manière concrète les contextes, les interventions, les résultats à court, à moyen et à long terme et le but des programmes amis des aînés. Là encore, les concepts qu'ils ont définis à partir des programmes existants et à l'aide de données sur des villes européennes concordent fortement avec ce qui a été élaboré grâce au processus que nous avons suivi, ce qui renforce la valeur de nos résultats et montre la transférabilité de nos conclusions au contexte des autres pays occidentaux.

\section{Points forts et limites}

La démarche que nous décrivons dans cet article visait à produire un ensemble d'indicateurs adaptés au contexte canadien et à la fois acceptables et utiles pour les collectivités qui mettent en place des initiatives amies des aînés. Différentes méthodes ont donc été adoptées afin que notre démarche soit solide et de grande qualité. L'étape initiale - la revue de la littérature et la détermination d'indicateurs potentiels a fait appel à des méthodes qualitatives. La crédibilité, la transférabilité, la fiabilité et la constance interne de cette première étape étaient essentielles à notre démarche ${ }^{56}$.

- La crédibilité des analyses s'appuie sur l'expertise de l'équipe de recherche en matière d'analyses qualitatives, l'examen du codage réalisé par l'équipe de recherche et la définition par consensus des concepts à regrouper en un indicateur.

- La transférabilité à d'autres pays comparables à ceux décrits dans notre revue de la documentation est probable. Toutefois, les indicateurs déterminés ne sont pas transférables à des pays non occidentaux ou à des pays à revenu intermédiaire ou faible. 
- La fiabilité est soutenue par la description de nos méthodes d'identification des concepts d'indicateur au moyen de démarches déductives et inductives.

- La constance interne repose sur la triangulation, la participation de plusieurs chercheurs en recherche qualitative à la revue de la documentation et la prise de notes détaillées tout au long de la détermination et de la simplification des listes initiales d'indicateurs.

Plusieurs facteurs restreignent la possibilité de généraliser les conclusions des deux sondages de consultation. Tout d'abord, les consultations ont été menées auprès d'intervenants reconnus mais qui ne forment peut-être pas un échantillon représentatif des personnes intéressées par les questions relatives aux aînés ou l'adaptation à leurs besoins. Nous n'avons par exemple pas consulté la population en général, et les intervenants connaissaient dans une mesure plus ou moins étendue les initiatives amies des aînés. Peu de répondants avaient plus de 75 ans, et peu d'entre eux ont indiqué appartenir à une minorité visible. Notre échantillon ne comprenait pas de membres de la société les plus vulnérables et les plus marginalisés, en particulier les personnes itinérantes, à faible revenu, atteintes de démence, vivant en établissement ou n'ayant pas accès à Internet.

En outre, les taux de réponse à chacun des sondages étaient plutôt faibles et, en conséquence, le nombre final de répondants s'est révélé limité. Enfin, une tendance à la lassitude a été remarquée chez les répondants : la proportion de données manquantes augmentait vers la fin des deux sondages, et quelques participants ont indiqué que les sondages étaient trop longs. Les réponses ont été examinées visuellement en vue de repérer d'éventuels biais dans les réponses (par exemple, des répondants qui inscriraient la même réponse à toutes les questions), mais aucune tendance n’a émergé à cet égard.

Le fait que les définitions des deux critères (capacité d'être mis en pratique et faisabilité) incluaient plusieurs concepts a pu entraîner de la confusion chez certains répondants du second sondage et a été une source d'incertitude dans l'interprétation des résultats. Si un répondant a inscrit qu'un indicateur avait une " capacité élevée d'être mis en œuvre ", on ne sait pas avec certitude si c'est parce qu'il estimait que l'ordre de gouvernement approprié était impliqué ou parce que l'indicateur pouvait s'adapter à un changement de politique, à un nouveau programme ou à une nouvelle activité. De même, si un indicateur a été évalué comme étant " très faisable ", le répondant voulait-il dire que les données étaient à la fois qualitatives et quantitatives, ou que les données pouvaient être recueillies facilement? Au cours de consultations futures, ces critères devraient être séparés afin qu'un seul élément soit évalué par question.

\section{Conclusion}

De plus en plus de collectivités mettent en place des projets amis des aînés à l'échelle du Canada, et bon nombre d'entre elles sont prêtes à procéder à l'évaluation de leurs activités. Il existe un vif intérêt à partager des outils de mesure des progrès réalisés dans l'adaptation aux besoins des aînés, ce qui constitue le cinquième jalon pancanadien des CAA. Dans cet article, nous avons décrit la démarche suivie par l'ASPC dans le but de définir et de sélectionner des indicateurs potentiels pour les CAA. À partir d'une liste initiale de 241 indicateurs, nous sommes parvenus à une liste de 43 indicateurs, sur la base de leur capacité à être mis en pratique et de leur faisabilité.

\section{Remerciements}

Nous aimerions souligner les contributions de Danielle Maltais et de Dawn Nickel à ce projet.

\section{Références}

1. Organisation mondiale de la Santé. Guide mondial des villes-amies des aînés. Genève $(\mathrm{CH})$ : Organisation mondiale de la Santé; 2007.

2. Ministres fédéral, provinciaux et territoriaux responsables des aînés. Initiative des collectivités rurales et éloignées amies des aînés : un guide. Ottawa (Ont.) : Agence de la santé publique du Canada; 2009.

3. Agence de la santé publique du Canada. Collectivités-amies des aînés [Internet]. Ottawa (Ont.) : Agence de la santé publique du Canada; 2015 [mise à jour le 29 mars 2016; consultation le 24 avril 2016]. Consultable en ligne à la page : http:// www.phac-aspc.gc.ca/seniors-aines/ afc-caa-fra.php
4. Bauer G, Davies JK, Pelikan J, Noack H, Broesskamp U, Hill C. Advancing a theoretical model for public health and health promotion indicator development: proposal from the EUHPID consortium. Eur J Public Health. 2003;13(suppl 3):107-113.

5. Brown D. Good practice guidelines for indicator development and reporting. Third World Forum on 'Statistics, Knowledge and Policy' Charting Progress, Building Visions, Improving Life, 2009 Oct 27-30, Busan, Korea. Wellington (NZ) : Statistics New Zealand; 2009.

6. Betancourt MT, Roberts KC, Bennett TL, Driscoll ER, Jayaraman G, Pelletier L. Surveillance des maladies chroniques au Canada : Cadre conceptuel d'indicateurs des maladies chroniques. Maladies chroniques et blessures au Canada. 2014;34 suppl. 1:1-33.

7. Beaulieu M, Garon S. Le projet «Villes amies des aînés » de l'OMS : un modèle international ayant fait l'objet d'une étude pilote à Sherbrooke, Québec - Quelques constats pour améliorer les conditions de vie des Québécois aînés dans nos villes. Mémoire présenté à la consultation publique sur les conditions de vie des aînés. Québec (Qc) : Gouvernement du Québec; 2007.

8. Menec V, Button C, Blandford A. Agefriendly communities in Manitoba. Report on survey findings. Winnipeg (MB) : Centre on Aging, University of Manitoba; 2008.

9. Ville d'Ottawa. Résultats des consultations du sommet sur les aînés [Internet]. Ottawa (Ont.) : Ville d'Ottawa; [consultation le 24 avril 2016]. Consultable en ligne à la page : http://ottawa.ca/fr/resultats-des -consultations

10. Wiley M. Niagara age-friendly community initiative: year 1. 2010-2011 evaluation report. St. Catharines (Ont.) : Niagara Research and Planning Council; 2011.

11. Saskatoon Council on Aging. Agefriendly Saskatoon initiative: findings report. Saskatoon (SK) : Saskatoon Council on Aging; 2011. 
12. AdvantAge Initiative. Indicators list: essential elements of an elder friendly community [Internet]. New York (NY) : The AdvantAge Initiative; 2015 [consultation le 24 avril 2016]. PDF téléchargeable à partir du lien : http://www.vnsny.org/advantage /indicators.pdf

13. Kihl M, Brennan D, Gabhawala N, List J, Mittal P. Livable communities: an evaluation guide. Washington (DC) : AARP; 2005.

14. Clemson L, Manor D, Fitzgerald M. Behavioral factors contributing to older adults falling in public places. OTJR. 2003;23(3):107-117. doi: 10.1177 /153944920302300304.

15. Gallagher N, Gretebeck KA, Robinson JC, Torres ER, Murphy SL, Martyn KK. Neighborhood factors relevant for walking in older, African American adults. J Aging Phys Act. 2010;18(1): 99-115.

16. Hoehner C, Ivy A, Ramirez LK, Handy S, Brownson RC. Active neighborhood checklist: a user-friendly and reliable tool for assessing activity friendliness. Am J Health Promot. 2007;21(6): 534-537.

17. Hooker SP, Cirill L, Wicks L. Walkable neighborhoods for seniors: the Alameda County experience. J Appl Gerontol. 2007;26:157-181. doi: 10.1177 /0733464807299997.

18. Michael YL, Green MK, Farquhar SA. Neighborhood design and active aging. Health Place. 2006;12:734-740.

19. Resnick B, King A, Riebe D, Ory M. Measuring physical activity in older adults: use of the Community Health Activities Model Program for Seniors Physical Activity Questionnaire and the Yale Physical Activity Survey in three behavior change consortium studies. West J Nurs Res. 2008;30(6): 673-689. doi: 10.1177/0193945907311320.

20. Shendell DG, Johnson ML, Sanders DL et collab. Community built environment factors and mobility around senior wellness centers: the concept of "safe senior zones". J Environ Health. 2011;73(7):9-18.
21. National Association of Area Agencies et collab. The maturing of America: getting communities on track for an aging population. Washington (DC) : National Association of Area Agencies on Aging; 2006.

22. Creating an age-friendly NYC one neighborhood at a time: a toolkit for establishing an age-friendly neighborhood in your community. New York (NY) : The New York Academy of Medicine; 2012. Joint publication of the Age-Friendly NYC.

23. Thomas P. Let us in : why southern Nevada needs visitable homes. Desert Companion. 2011 Jan 27-30;62.

24. Modlich R. Age-friendly communities: a women's issue. Women and Environments International Magazine. 2011;84/85.

25. Spinks A, Turner C, Nixon J, McClure R. The 'WHO Safe Communities' model for the prevention of injury in whole populations. London (UK) : The Cochrane Collaboration, John Wiley and Sons Ltd; 2009.

26. Arrif T, Rioux L. Les pratiques des espaces verts urbains par les personnes âgées. L'exemple du parc de Bercy. Pratiques psychologiques. 2011; 17:5-17.

27. Pochet P, Corget R. Entre " automobilité », proximité et sédentarité, quels modèles de mobilité quotidienne pour les résidents âgés des espaces périurbains? Espace Populations Sociétés. 2010;1:69-81.

28. Broome K, Nalder E, Worrall L, Boldy D. Age-friendly buses? A comparison of reported barriers and facilitators to bus use for older and younger adults. Australas J Ageing. 2010;29(1):33-38.

29. Everingham J, Petriwskyj A, Warburton $\mathrm{J}$, Cuthill $\mathrm{M}$, Bartlett $\mathrm{M}$. Information provision for an age-friendly community. Ageing Int. 2009;34:79-98. doi: 10.1007/s12126-009-9036-5.

30. Lindqvist K, Timpka T, Schelp L. Evaluation of an inter-organizational prevention program against injuries among the elderly in a WHO Safe Community. Public Health. 2001;115: 308-16.
31. Wennberg H, Hyden C, Stahl A. Barrier-free outdoor environments: older peoples' perceptions before and after implementation of legislative directives. Transp Policy. 2010;17(6): 464-74. doi: 10.1016/j.tranpol.2010.04 .013 .

32. McGarry P, Morris J. A great place to grow older: a case study of how Manchester is developing an agefriendly city. Working with Older People. 2011;15(1):38-46.

33. Doyle V, Gallagher E. Creating an agefriendly business in BC. Vancouver (BC) : BC Chamber of Commerce; 2011.

34. Gerotech Research Associates. Agefriendly British Columbia: lessons learned from October, 2007 September, 2010. Submitted to Seniors Healthy Living Secretariat, Ministry of Healthy Living and Sport. Victoria, BC : Gerotech Research Associates; 2010.

35. Mahaffey R. Planning for the future: age-friendly and disability-friendly official community plans. Richmond (BC) : Union of BC Municipalities; 2010.

36. Provincial Health Services Authority. Indicators for a healthy built environment in BC. Vancouver (BC) : Provincial Health Services Authority; 2008.

37. Stepaniuk JA, Tuokko H, McGee P, Garrett DD, Benner EL. Impact of transit training and free bus pass on public transportation use by older drivers. Prev Med. 2008;47(3):335-337.

38. Gagnon L, Savoie A. Rapport de la consultation publique sur les conditions de vie des aînés : Préparons l'avenir avec nos aînés. Québec (Qc) : Gouvernement du Québec, ministère de la Famille et des Aînés; 2008.

39. Martin V. Municipalité amie des aînés. Favoriser le vieillissement actif au Québec. Québec (Qc) : Gouvernement du Québec, ministère de la Famille et des Aînés; 2009.

40. Ministère de la Famille et des Aînés. Municipalité amie des aînés. Programme de soutien. Québec (Qc) : Gouvernement du Québec; 2012. 
41. Rochman J, Tremblay DG. Le soutien à la participation sociale des aînés et le programme "ville amie des aînés » au Québec. Note de recherche de l'Alliance de recherche université-communauté sur la gestion des âges et des temps sociaux, Télé-université/Université de Québec à Montréal. Montréal (Qc) : Université du Québec; 2010.

42. Lockett D, Willis A, Edwards N. Through seniors' eyes: an exploratory qualitative study to identify environmental barriers to and facilitators of walking. Revue canadienne de recherche en sciences infirmières. 2005; 37(3):48-65.

43. Ministère des Affaires municipales et du Logement et l'Institut des planificateurs professionnels de l'Ontario. L’aménagement créatif. Toronto (Ont.) : Institut des planificateurs professionnels de l’Ontario; 2009.

44. Lee KK. Fit cities: how active design can build healthier and more sustainable communities. Vancouver (BC) : Active Design Leadership Institute; 2010. PDF téléchargeable à partir du lien : http://www.ncchpp.ca/docs /CLASP_24Nov2010_KarenLee.pdf

45. Menec VH, Means R, Keating N, Parkhurst G, Eales J. Conceptualizing age-friendly communities. La Revue canadienne du vieillissement. 2011;30: 479-493.

46. Agence de la santé publique du Canada. Pour bien communiquer avec les aînés : faits, conseils et idées. Ottawa (Ont.) : Agence de la santé publique du Canada; 2010.

47. Société canadienne d'hypothèques et de logement. Indicateurs propres au vieillissement de la population à l'intention des collectivités. Le Point en recherche. Ottawa (Ont.) : Société canadienne d'hypothèques et de logement; 2008.

48. Hayes MV, Willems SM. Healthy community indicators: the perils of the search and the paucity of the find. Health Promot Int. 1990;5(2):161-166. doi: $10.1093 /$ heapro/5.2.161.

49. Boyatzis RE. Transforming qualitative information : thematic analysis and code development. Thousand Oaks (CA) : Sage; 1998.
50. World Health Organization AgeFriendly Indicator Development Group. Meeting summary: Developing indicators for the global age-friendly cities. August 30-31, 2012; St. Gallen (CH). Geneva (CH) : WHO; 2012.

51. Équipe de développement des indicateurs de blessure chez les enfants et les jeunes au Canada. Mesurer les blessures est important : indicateurs de blessure chez les enfants et les jeunes au Canada, volume 1. Vancouver (C.-B.) : British Columbia Injury Research and Prevention Unit; 2010.

52. Daniel M. Rating health and social indicators for use with indigenous communities: a tool for balancing cultural and scientific utility. Soc Indic Res. 2009;(94):241-256.

53. USAID Center for Development Information and Evaluation. Selecting performance indicators. Performance monitoring and evaluation TIPS. Washington (DC) : United States Agency for International Development; 1996;(6):1-4.

54. Organisation mondiale de la Santé. Mesurer la convivialité des villes à l'égard des aînés : guide pour l'utilisation d'indicateurs de base. Genève (CH) : OMS; 2015.

55. Jackisch J, Zamaro G, Green G, Huber M. Is a healthy city also an agefriendly city? Health Promot Int. 2015;30 Suppl 1:i108-117. doi : 10.1093 /heapro/dav039.

56. Lincoln YS, Guba EG. Naturalistic Inquiry. Newbury Park (CA) : Sage Publications; 1985. 